\begin{tabular}{|c|c|c|}
\hline & Int.J.Curr.Microbiol.App.Sci (2021) 10(10): 118-128 & \\
\hline$\frac{}{\text { EXCELLENT }}$ & $\begin{array}{l}\text { International Journal of Current Microbiology and Applied Sciences } \\
\text { ISSN: 2319-7706 Volume } 10 \text { Number } \mathbf{1 0}(\mathbf{2 0 2 1 )} \\
\text { Journal homepage: http://www.ijcmas.com }\end{array}$ & 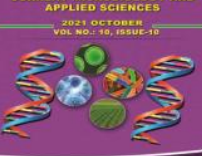 \\
\hline PUBLISHERS & & wwwijcmas.com \\
\hline
\end{tabular}

\title{
Combining Ability Analysis for Seed Cotton Yield and Yield Contributing Traits in Desi Cotton (Gossypium arboreum L.) over the Environments
}

\author{
V. N. Chinchane*, D. B. Deosarkar, K. S. Baig and H. V. Kalpande \\ Department of Agricultural Botany, Genetics and Plant Breeding, Vasantrao Naik \\ Marathawada Krishi Vidyapeeth, Parbhani - 431402, M.S., India \\ *Corresponding author
}

\section{A B S T R A C T}

Keywords

Combining ability, GCA, SCA, yield, harvest index

Article Info

Accepted: 05 September 2021 Available Online: 10 October 2021
Cotton is one of the most important fiber and cash crop of India and plays a dominant role in the industrial and agricultural economy of the country. Fifty six crosses with fifteen parents and four checks viz., PKVDH 1, PKVSuvarna, NACH 12 and PA 255 were grown in Randomized Block Design with two replications. Pooled over the environments, the highest GCA effect for seed cotton yield plant was showed by line PAIG 346and also exhibited high GCA (in desirable direction) for the traits, sympodia per plant, number of bolls per plant, seed index, lint index and harvest index. Among the testers, highest GCA for seed cotton yield per plant (9.72) was reported in CNA 449.This tester showed significantly desirable GCA for the characters viz. plant height, number of sympodia per plant, number of bolls per plant, boll weight, seed index and harvest index. Pooled over the environments, among the crosses highest SCA for seed cotton yield per plant was reported in PA 740 x Digvijay. It has also exhibited significant SCA in desirable direction for days to $50 \%$ boll bursting, number of sympodia per plant, number of bolls per plant, boll weight, seed index and harvest index. Another cross which ranked second for with high per se and high SCA for seed cotton yield per plant was PAIG 346 x DWDa 1402.It showed significantly desirable SCA for days to $50 \%$ flowering, number of sympodia per plant, number of bolls per plant, seed index, lint index and harvest index.

\section{Introduction}

Cotton, the king of fibres, occupies a preeminent position as a commercial crop in India. Cotton also known as 'white gold' as it is preferred by farmers as cash crop beside other field crops. It is grown commercially in the temperate and tropical regions of more than 70 countries. India is perhaps the first country to make use of cotton. Cotton, the 'white gold' enjoys a pre-eminent status among all cash crops in the country.

It is grown commercially in the temperate and tropical regions of more than 70 countries. Specific areas of production include countries 
such as China, USA, India, Pakistan, Uzbekistan, Turkey, Australia, Greece, Brazil, Egypt etc. Genetic improvement in desi cotton could be gained either through selection or exploitation of specific hybrid.

Therefore, more emphasis should be given to increase the seed cotton yield per unit area by developing hybrids with short stature, big boll size, longer staple length with sustained yield in multiple environments.

To achieve such desirable characteristics in a new cultivar, proper breeding strategies should be followed.

There is an urgent need to promote those cottons that could come closer in quality to the most sought by modern textile mills.

Line $\mathrm{x}$ tester analysis provides a systematic approach for selection of appropriate parents on the basis of general combining ability effects (GCA) and superior crosses on the basis of specific combining ability effects (SCA).

Combining ability describes the breeding value of parental lines to produce hybrids. Sprague and Tatum (1942) used the terms general combining ability (GCA) to designate the average performance of a line in hybrid combinations and specific combining ability (SCA) as deviation in performance of across combination from that predicted on the basis of the general combining abilities of the parents involved in the cross.

In order to choose appropriate parents for hybridization as well as specific crosses, it is essential to determine the combining ability of parents and specific combining ability (SCA) of cross combinations by using line $\mathrm{x}$ tester analysis. The method has been widely used by plant breeders. This method was applied to improve self and cross-pollinated crop plants.

\section{Materials and Methods}

The present study comprised of seven females (lines) and eight males (testers) with four standard checks thus making $56 \quad \mathrm{~F}_{1} \mathrm{~s}$ using Line $\mathrm{x}$ Tester mating design. These lines, testers and hybrids along with four checks were sown during kharif, 2016 at three locations viz., Cotton Research Station, Nanded (L-1), Experimental farm of Department of Agricultural Botany, VNMKV, Parbhani (L-2) and Experimental farm, Agricultural Research Station, Badnapur(L-3).

The observations were recorded on days to 50 $\%$ flowering, days to $50 \%$ boll bursting, plant height $(\mathrm{cm})$, number of sympodia per plant, number of boll per plant, boll weight $(\mathrm{g})$, seed cotton yield per plant $(\mathrm{g})$, seed index $(\mathrm{g})$, lint index (g) and harvest index (\%).Combining ability analysis was based on the procedure developed by Kempthorne (1957) related to design II of Comstock and Robinson (1952).

\section{Results and Discussion}

The pooled analysis of variance for line $\mathrm{x}$ tester was found significant for most of the characters. The significance of environment $\mathrm{x}$ crosses interaction for days to $50 \%$ boll bursting, plant height, number of sympodia per plant, number of bolls per plant, boll weight, seed cotton yield per plant and days to maturity given the diversity between the environments sampled. Environment $x$ line effect were found significant for plant height and ginning percentage whereas the environment $\mathrm{x}$ tester effect were found significant for number of sympodia per plant, boll weight and seed cotton yield per plant. This significance indicated the significant differences between the additive genetic contributions of individual line or tester to overall genetic variation observed between the crosses. The significance of environment $\mathrm{x}$ line $\mathrm{x}$ tester interaction effect for days to $50 \%$ 
boll bursting, number of sympodia per plant, number of bolls per plant, boll weight, seed cotton yield per plant, seed index and days to maturity indicated higher interaction of nonadditive genetic effects for yield with environments. This could be the reason for difference in heterosis over the environment for yield.

\section{GCA effect of parents}

Pooled over the environments, the highest GCA effect for seed cotton yield plant was showed by line PAIG 346 (6.04). This line has also showed high GCA in desirable direction number of sympodia per plant, number of bolls per plant, seed index, lint index and harvest index. Another line with high GCA which also showed high per se for seed cotton yield was PA 809. This genotype also showed the higher GCA in desirable direction for number of sympodia per plant, number of bolls per plant, seed index and harvest index. Line PA 809 found to be the best general combiner for yield and yield contributing characters which in combination can be efficiently used for most of the yield improvement. Line PA 812 was found to be the best general combiner for earliness traits viz. days to $50 \%$ flowering, days to $50 \%$ boll bursting, days to maturity.

Among the testers, highest GCA for seed cotton yield per plant (9.72) was reported in CNA 449. This tester showed significantly desirable GCA for the characters viz. plant height, number of sympodia per plant, number of bolls per plant, boll weight, seed index and harvest index. Hence this tester could be used for improving yield in the cotton. Second largest GCA for seed cotton yield per plant was recorded by the tester AKA 8 (6.12). This genotype also showed the higher GCA in desirable direction for earliness characters viz. days to $50 \%$ flowering, days to $50 \%$ boll bursting, days to maturity as well as for number of sympodia per plant, number of bolls per plant and harvest index. Other testers which showed high GCA for seed cotton yield plant were JLA 794 (4.44) and DWDa 1402 (4.03). These testers also showed significant desirable GCA for plant height, number of sympodia per plant, number of bolls per plant, boll weight, seed index and harvest index. Therefore, these testers could be used in the improvement of the said traits. Almost identical results have been reported by Deshpande et al., (2003), Maisuria et al., (2006), Laxman (2010), Kumar et al., (2014), Madhuri et al., (2014), Patel and Choudhari (2015) and Shakeel et al., (2016).

\section{SCA effect of crosses}

Pooled over the environments, among the crosses highest SCA for seed cotton yield per plant was reported in PA 740 x Digvijay (15.53). It has also exhibited significant SCA in desirable direction for days to $50 \%$ boll bursting, number of sympodia per plant, number of bolls per plant, boll weight, seed index and harvest index. Another cross which ranked second for with high per se and high SCA for seed cotton yield per plant was PAIG 346 x DWDa 1402 (11.92). It showed significantly desirable SCA for days to $50 \%$ flowering, number of sympodia per plant, number of bolls per plant, seed index, lint index and harvest index. Cross PA $809 \times$ AKA 8 ranked third with respect to high per se and high SCA (11.76). It recorded significantly desirable SCA for number of sympodia per plant, number of bolls per plant, harvest index and days to maturity. It also recorded nonsignificant SCA in desirable direction for days to $50 \%$ flowering, days to $50 \%$ boll bursting, plant height, seed index and harvest index. In the present study, cross combinations viz. PAIG 346 x JLA 794, PAIG 346 x DWDa 1402 and PA $809 \times$ AKA 8 possessed high per se, SCA with highly significant GCA of both the parents. 
Table.1 Pooled estimates of GCA effects of parents and SCA effects of crosses studied over three environments

\begin{tabular}{|c|c|c|c|c|c|c|c|c|c|}
\hline $\begin{array}{l}\text { Sr. } \\
\text { No. }\end{array}$ & Genotype & $\begin{array}{c}\text { Days to } 50 \\
\% \\
\text { flowering }\end{array}$ & $\begin{array}{c}\text { Days to } 50 \\
\% \text { boll } \\
\text { bursting }\end{array}$ & $\begin{array}{c}\text { Plant } \\
\text { height } \\
\text { (cm) }\end{array}$ & $\begin{array}{l}\text { Number of } \\
\text { sympodia } \\
\text { /plant }\end{array}$ & $\begin{array}{l}\text { Number of } \\
\text { bolls /plant }\end{array}$ & $\begin{array}{l}\text { Boll weight } \\
\text { (g) }\end{array}$ & $\begin{array}{l}\text { Seed cotton } \\
\text { yield /plant } \\
\text { (g) }\end{array}$ & $\begin{array}{l}\text { Seed index } \\
\text { (g) }\end{array}$ \\
\hline & GCA Lines & & & & & & & & \\
\hline 1 & PA 801 & $0.40 *$ & $0.79 * *$ & $1.46^{*}$ & -0.56 & 0.14 & -0.003 & 0.37 & $-0.14 * *$ \\
\hline 2 & PA 740 & $0.40 *$ & 0.37 & 0.72 & -0.63 & $-1.13 * *$ & $0.06^{* *}$ & $-2.18 * *$ & $0.06^{* *}$ \\
\hline 3 & PA 812 & $-1.64 * *$ & $-1.53 * *$ & $-3.64 * *$ & -0.50 & -0.34 & $-0.03 *$ & 0.10 & $0.08 * *$ \\
\hline 4 & PA 809 & 0.31 & 0.33 & $1.50 *$ & $0.81 *$ & $2.09 * *$ & 0.02 & $3.81 * *$ & $0.06^{*}$ \\
\hline 5 & PA 785 & 0.21 & 0.37 & $-2.72 * *$ & 0.30 & 0.49 & $-0.05 * *$ & 0.61 & $-0.24 * *$ \\
\hline 6 & PA 832 & $-0.62 * *$ & $-0.56 * *$ & 0.15 & $-2.24 * *$ & $-3.34 * *$ & $-0.05 * *$ & $-8.75 * *$ & 0.02 \\
\hline \multirow[t]{2}{*}{7} & PAIG 346 & $0.92 * *$ & 0.21 & $2.51 * *$ & $2.82 * *$ & $2.09 * *$ & $0.06^{* *}$ & $6.04 * *$ & $0.21 * *$ \\
\hline & GCA Testers & & & & & & & & \\
\hline 1 & AKA 8 & $-2.98 * *$ & $-3.39 * *$ & -1.05 & $1.50 * *$ & $2.88 * *$ & $-0.07 * *$ & $6.12 * *$ & $-0.21 * *$ \\
\hline 2 & PhuleDhanwantary & 0.27 & 0.10 & $-16.61 * *$ & $-3.42 * *$ & $-3.74 * *$ & $-0.10 * *$ & $-10.25 * *$ & $-0.52 * *$ \\
\hline 3 & CNA 449 & $0.87 * *$ & $1.17 * *$ & $6.35 * *$ & $2.87 * *$ & $3.45 * *$ & $0.08 * *$ & $9.72 * *$ & $0.40 * *$ \\
\hline 4 & HD 514 & $-2.50 * *$ & $-3.042 * *$ & $-9.76 * *$ & $1.56 * *$ & $1.90 * *$ & $-0.04 * *$ & $3.52 * *$ & $0.28 * *$ \\
\hline 5 & DWDa 1402 & $-0.79 * *$ & $-0.94 * *$ & $7.92 * *$ & $0.76^{*}$ & $1.32 * *$ & $0.06^{* *}$ & $4.03 * *$ & -0.002 \\
\hline 6 & JLA 794 & $0.56 * *$ & $0.76 * *$ & $8.95 * *$ & 0.38 & $1.44 * *$ & $0.09 * *$ & $4.44 * *$ & $0.54 * *$ \\
\hline 7 & Digvijay & $2.06 * *$ & $2.50 * *$ & $2.90 * *$ & $-2.18 * *$ & $-4.71 * *$ & $-0.03^{*}$ & $-10.43 * *$ & 0.005 \\
\hline \multirow[t]{2}{*}{8} & G.Cot 23 & $2.51 * *$ & $2.83 * *$ & 1.29 & $-1.47 * *$ & $-2.53 * *$ & 0.02 & $-7.14 * *$ & 0.005 \\
\hline & SCA Crosses & & & & & & & & \\
\hline 1 & PA 801 x AKA 8 & $-3.80 * *$ & $-4.08 * * *$ & $-4.50 *$ & 0.56 & -0.09 & 0.04 & 1.73 & -0.12 \\
\hline 2 & $\begin{array}{c}\text { PA } 801 \text { x } \\
\text { PhuleDhanwantary }\end{array}$ & $-2.40 * *$ & $-1.74 * *$ & -2.00 & -0.18 & -0.33 & $0.09 * *$ & 2.47 & 0.02 \\
\hline 3 & PA 801 x CNA 449 & 0.17 & 0.18 & -3.26 & -1.51 & -1.95 & 0.05 & -1.44 & 0.08 \\
\hline 4 & PA 801 x HD 514 & 0.55 & 0.39 & $15.24 * *$ & 1.93 & $4.56^{* *}$ & 0.04 & $7.96 * *$ & -0.14 \\
\hline 5 & PA 801 x DWDa 1402 & $-2.16 * *$ & $-2.86 * *$ & 0.49 & 1.00 & 0.64 & 0.02 & -0.28 & -0.11 \\
\hline 6 & PA 801 x JLA 794 & -0.52 & -0.91 & 1.16 & 1.41 & 1.75 & -0.05 & 1.81 & -0.002 \\
\hline 7 & PA 801 x Digvijay & $4.81 * *$ & $5.01 * *$ & $-4.22 *$ & -1.69 & -1.42 & $-0.08^{*}$ & $-5.35^{*}$ & 0.05 \\
\hline 8 & PA 801 x G.Cot 23 & $3.36^{* *}$ & $4.01 * *$ & -2.90 & -1.53 & $-3.17 * *$ & $-0.10 * *$ & $-6.90 * *$ & $0.22 *$ \\
\hline 9 & PA $740 \times$ AKA 8 & $1.69 * *$ & $2.50 * *$ & 2.04 & $-3.60 * *$ & -1.57 & $-0.08 *$ & $-6.41 * *$ & $-0.38 * *$ \\
\hline 10 & $\begin{array}{c}\text { PA } 740 \text { x } \\
\text { PhuleDhanwantary }\end{array}$ & 0.43 & $2.33 * *$ & -0.49 & 0.72 & $-2.45^{*}$ & -0.03 & -4.24 & -0.14 \\
\hline
\end{tabular}


Table.1 (Contd...)

\begin{tabular}{|c|c|c|c|c|c|c|c|c|c|}
\hline $\begin{array}{l}\text { Sr. } \\
\text { No. }\end{array}$ & Genotype & $\begin{array}{l}\text { Days to } 50 \\
\text { \% flowering }\end{array}$ & $\begin{array}{c}\text { Days to } 50 \\
\% \text { boll } \\
\text { bursting }\end{array}$ & $\begin{array}{l}\text { Plant height } \\
\text { (cm) }\end{array}$ & $\begin{array}{l}\text { Number of } \\
\text { sympodia } \\
\text { /plant }\end{array}$ & $\begin{array}{l}\text { Number of } \\
\text { bolls /plant }\end{array}$ & $\begin{array}{c}\text { Boll weight } \\
\text { (g) }\end{array}$ & $\begin{array}{c}\text { Seed cotton } \\
\text { yield /plant } \\
\text { (g) }\end{array}$ & $\begin{array}{c}\text { Seed index } \\
\text { (g) }\end{array}$ \\
\hline 11 & PA 740 x CNA 449 & 0.17 & 0.09 & 0.34 & -1.58 & $-3.54 * *$ & $-0.07 *$ & $-5.45^{*}$ & -0.002 \\
\hline 12 & PA $740 \times$ HD 514 & 0.21 & 0.47 & -1.36 & -0.10 & $2.81 *$ & -0.05 & 4.44 & $-0.17 *$ \\
\hline 13 & PA 740 x DWDa 1402 & 0.00 & -0.11 & -0.43 & 0.24 & 0.22 & -0.01 & 0.14 & 0.10 \\
\hline 14 & PA 740 x JLA 794 & -0.85 & $-1.16^{*}$ & 0.54 & $-3.42 * *$ & $-5.26^{* *}$ & -0.008 & $-11.36 * *$ & -0.01 \\
\hline 15 & PA 740 x Digvijay & -0.85 & $-1.56 * *$ & -0.38 & $4.71 * *$ & $6.52 * *$ & $0.19 * *$ & $15.53 * *$ & $0.44 * *$ \\
\hline 16 & PA 740 x G.Cot 23 & -0.80 & $-2.56 * *$ & -0.23 & $3.03 * *$ & $3.27 * *$ & 0.05 & $7.35^{* *}$ & 0.16 \\
\hline 17 & PA 812 x AKA 8 & 0.06 & -0.08 & -0.49 & 0.001 & -0.77 & 0.05 & 1.41 & -0.03 \\
\hline 18 & PA 812 x PhuleDhanwantary & $-1.19 *$ & -1.08 & -0.76 & -0.64 & 0.96 & 0.01 & -1.06 & -0.02 \\
\hline 19 & PA $812 \times$ CNA 449 & -0.62 & -0.98 & -1.39 & -1.64 & -0.91 & -0.04 & -3.76 & -0.11 \\
\hline 20 & PA 812 x HD 514 & -0.24 & -0.27 & -1.44 & $2.70 * *$ & 1.89 & -0.006 & 3.27 & $0.34 * *$ \\
\hline 21 & PA 812 x DWDa 1402 & $1.87 * *$ & $2.13 * *$ & -2.20 & $-2.36^{*}$ & $-2.54 *$ & 0.02 & -3.75 & -0.09 \\
\hline 22 & PA 812 x JLA 794 & 0.52 & 0.58 & -0.22 & -0.18 & -2.16 & -0.01 & -3.51 & $-0.18^{*}$ \\
\hline 23 & PA 812 x Digvijay & -0.47 & -0.65 & $3.51^{*}$ & 0.21 & $2.40 *$ & 0.06 & 2.59 & $0.16^{*}$ \\
\hline 24 & PA 812 x G.Cot 23 & 0.06 & 0.34 & 2.99 & 1.90 & 1.15 & $-0.08 *$ & $4.81 *$ & -0.07 \\
\hline 25 & PA 809 x AKA 8 & 0.94 & -0.62 & -1.67 & $4.45^{* *}$ & $4.76 * *$ & 0.008 & $11.76^{* *}$ & 0.12 \\
\hline 26 & PA 809 x PhuleDhanwantary & -0.48 & -0.45 & 0.15 & -0.85 & -1.21 & -0.006 & -0.66 & $0.17 *$ \\
\hline 27 & PA 809 x CNA 449 & 0.25 & 0.64 & $5.59 * *$ & 0.14 & 1.03 & -0.04 & -2.38 & $0.21 *$ \\
\hline 28 & PA 809 x HD 514 & $0.96 *$ & 1.02 & $-4.82 * *$ & -0.84 & -0.62 & 0.008 & $-4.91 *$ & 0.15 \\
\hline 29 & PA 809 x DWDa 1402 & -0.41 & -0.74 & 2.42 & -1.44 & $-2.41 *$ & 0.02 & -2.12 & $0.19 *$ \\
\hline 30 & PA 809 x JLA 794 & -0.27 & -0.12 & 0.22 & -0.66 & -1.09 & 0.05 & -0.56 & 0.05 \\
\hline 31 & PA 809 x Digvijay & -0.10 & -0.36 & -3.22 & -1.13 & -2.30 & -0.06 & -2.53 & $-0.21^{*}$ \\
\hline 32 & PA 809 x G.Cot 23 & -0.89 & 0.64 & 1.32 & 0.33 & 1.85 & 0.02 & 1.40 & $-0.68 * *$ \\
\hline 33 & PA $785 \times$ AKA 8 & 0.21 & 0.17 & $3.62 *$ & -0.17 & -0.73 & -0.002 & -1.00 & $0.27 * *$ \\
\hline 34 & PA 785 x PhuleDhanwantary & 0.11 & 0.17 & 2.58 & 0.79 & $4.89 * *$ & 0.06 & $9.43 * *$ & $0.32 * *$ \\
\hline 35 & PA 785 x CNA 449 & 0.35 & 0.26 & -1.51 & 1.65 & $3.77 * *$ & 0.01 & $6.30 * *$ & -0.11 \\
\hline 36 & PA 785 x HD 514 & -0.76 & $-1.85 * *$ & -2.63 & -0.10 & $-5.38 * *$ & 0.05 & $-9.72 * *$ & -0.04 \\
\hline 37 & PA 785 x DWDa 1402 & $1.52 * *$ & $1.88 * *$ & 0.67 & -0.66 & -2.24 & -0.05 & $-4.83 *$ & $-0.41 * *$ \\
\hline 38 & PA 785 x JLA 794 & $1.00 *$ & $1.33^{*}$ & -1.37 & -0.36 & -0.02 & 0.009 & -1.99 & $-0.22 * *$ \\
\hline 39 & PA 785 x Digvijay & $-1.16^{*}$ & $-1.40 *$ & 0.29 & 0.88 & 0.80 & $-0.12 * *$ & 3.11 & $0.21 *$ \\
\hline
\end{tabular}


Table.1 (Contd...)

\begin{tabular}{|c|c|c|c|c|c|c|c|c|c|}
\hline $\begin{array}{l}\text { Sr. } \\
\text { No. }\end{array}$ & Genotype & $\begin{array}{c}\text { Days to } \\
50 \% \\
\text { flowering }\end{array}$ & $\begin{array}{c}\text { Days to } \\
50 \% \\
\text { boll } \\
\text { bursting }\end{array}$ & $\begin{array}{c}\text { Plant } \\
\text { height } \\
(\mathrm{cm})\end{array}$ & $\begin{array}{l}\text { Number } \\
\text { of } \\
\text { sympodia } \\
\text { /plant }\end{array}$ & $\begin{array}{l}\text { Number } \\
\text { of bolls } \\
\text { /plant }\end{array}$ & $\begin{array}{c}\text { Boll } \\
\text { weight } \\
\text { (g) }\end{array}$ & $\begin{array}{c}\text { Seed } \\
\text { cotton } \\
\text { yield } \\
\text { /plant }(\mathrm{g})\end{array}$ & $\begin{array}{c}\text { Seed } \\
\text { index } \\
(\mathrm{g})\end{array}$ \\
\hline 40 & PA 785 x G.Cot 23 & $-1.28 * *$ & 0.44 & -1.65 & -1.13 & -1.07 & 0.04 & -1.30 & -0.03 \\
\hline 41 & PA 832 x AKA 8 & 0.05 & 0.27 & 2.55 & -1.79 & -1.76 & 0.03 & $-7.41 * *$ & 0.08 \\
\hline 42 & $\begin{array}{c}\text { PA } 832 \mathrm{x} \\
\text { PhuleDhanwantary }\end{array}$ & 0.28 & -0.46 & 0.11 & $2.00 *$ & 0.13 & -0.06 & 1.16 & -0.08 \\
\hline 43 & PA 832 x CNA 449 & -0.14 & -1.08 & 0.88 & $3.47 * *$ & 2.12 & 0.03 & $6.79 * *$ & -0.14 \\
\hline 44 & PA 832 x HD 514 & $-1.92 * *$ & 0.15 & -1.00 & $-3.58 * *$ & $-3.95 * *$ & -0.06 & $-5.84 *$ & -0.07 \\
\hline 45 & PA 832 x DWDa 1402 & 0.35 & 0.44 & -0.82 & -0.71 & 0.86 & $0.07 *$ & -1.09 & 0.03 \\
\hline 46 & PA 832 x JLA 794 & 0.50 & 0.86 & -1.08 & -0.21 & $2.54 *$ & 0.02 & 3.90 & $0.24 * *$ \\
\hline 47 & PA 832 x Digvijay & -0.16 & -0.63 & 0.62 & 0.59 & -1.33 & $-0.07 *$ & -0.06 & $-0.24 * *$ \\
\hline 48 & PA 832 x G.Cot 23 & $1.04 *$ & 0.44 & -1.25 & 0.22 & 1.38 & 0.05 & 2.55 & $0.17 *$ \\
\hline 49 & PAIG $346 \times$ AKA 8 & 0.83 & $1.67 * *$ & -1.55 & 0.54 & 0.17 & -0.03 & -0.07 & 0.04 \\
\hline 50 & $\begin{array}{c}\text { PAIG } 346 \text { x } \\
\text { PhuleDhanwantary }\end{array}$ & $3.24 * *$ & 0.50 & 0.41 & -1.83 & -1.98 & $-0.07 *$ & $-7.10 * *$ & $-0.28 * *$ \\
\hline 51 & PAIG 346 x CNA 449 & -0.18 & 0.26 & -0.65 & -0.53 & -0.50 & 0.06 & -0.07 & 0.08 \\
\hline 52 & PAIG 346 x HD 514 & $1.19 *$ & $1.31 *$ & $-3.97 *$ & 0.88 & 0.69 & 0.01 & $4.80 *$ & -0.09 \\
\hline 53 & PAIG 346 x DWDa 1402 & $-1.18^{*}$ & -0.44 & -0.12 & $3.92 * *$ & $5.46 * *$ & -0.06 & $11.92 * *$ & $0.28 * *$ \\
\hline 54 & PAIG 346 x JLA 794 & -0.37 & -0.16 & 0.75 & $3.42 * *$ & $4.24 * *$ & -0.003 & $11.71 * *$ & 0.13 \\
\hline 55 & PAIG 346 x Digvijay & $-2.04 * *$ & $-1.90 * *$ & 3.39 & $-3.58 * *$ & $-4.67 * *$ & $0.07 *$ & $-13.29 * *$ & $-0.41 * * *$ \\
\hline \multirow[t]{5}{*}{56} & PAIG 346 x G.Cot 23 & $-1.49 * *$ & $-1.23 *$ & 1.74 & $-2.82 * *$ & $-3.42 * *$ & 0.02 & $-7.90 * *$ & $0.24 * *$ \\
\hline & S.E. \pm & & & & & & & & \\
\hline & Lines & 0.17 & 0.20 & 0.62 & 0.36 & 0.41 & 0.011 & 0.81 & 0.029 \\
\hline & Tester & 0.18 & 0.22 & 0.66 & 0.38 & 0.44 & 0.012 & 0.87 & 0.032 \\
\hline & Crosses & 0.49 & 0.58 & 1.76 & 1.01 & 1.17 & 0.032 & 2.30 & 0.084 \\
\hline
\end{tabular}


Table.1 (Contd...)

\begin{tabular}{|c|c|c|c|c|c|}
\hline $\begin{array}{l}\text { Sr. } \\
\text { No. }\end{array}$ & Genotype & $\begin{array}{l}\text { Lint index } \\
\text { (g) }\end{array}$ & $\begin{array}{l}\text { Harvest index } \\
(\%)\end{array}$ & $\begin{array}{c}\text { Ginning outturn } \\
(\%)\end{array}$ & Days to maturity \\
\hline & GCA Lines & & & & \\
\hline 1 & PA 801 & 0.01 & $-0.56 *$ & -0.31 & $0.83 * *$ \\
\hline 2 & PA 740 & -0.001 & 0.15 & $0.43 * *$ & 0.25 \\
\hline 3 & PA 812 & 0.02 & $-0.62 * *$ & $-0.40 * *$ & $-1.20 * *$ \\
\hline 4 & PA 809 & -0.03 & $0.66 * *$ & $0.55 * *$ & 0.33 \\
\hline 5 & PA 785 & 0.02 & -0.31 & -0.09 & 0.08 \\
\hline 6 & PA 832 & $-0.09 * *$ & $-1.39 * *$ & $-0.60 * *$ & $-0.89 * *$ \\
\hline \multirow[t]{2}{*}{7} & PAIG 346 & $0.06 * *$ & $2.07 * *$ & $0.42 *$ & $0.58 *$ \\
\hline & GCA Testers & & & & \\
\hline 1 & AKA 8 & 0.02 & $0.91 * *$ & $1.00 * *$ & $-2.54 * *$ \\
\hline 2 & PhuleDhanwantary & $-0.06 * *$ & $-1.87 * *$ & 0.02 & $-0.80 * *$ \\
\hline 3 & CNA 449 & $-0.05 *$ & $2.28 * *$ & $-0.52 * *$ & $1.33 * *$ \\
\hline 4 & HD 514 & $0.17 * *$ & $0.83 * *$ & $1.67 * *$ & $-2.71 * *$ \\
\hline 5 & DWDa 1402 & $-0.09 * *$ & 0.42 & $-1.06 * *$ & $-1.21 * *$ \\
\hline 6 & JLA 794 & -0.03 & $0.62 *$ & $-0.40 *$ & 0.54 \\
\hline 7 & Digvijay & -0.02 & $-1.55 * *$ & $-0.81 * *$ & $2.21 * *$ \\
\hline \multirow[t]{2}{*}{8} & G.Cot 23 & $0.06 * *$ & $-1.65 * *$ & 0.09 & $2.97 * *$ \\
\hline & SCA Crosses & & & & \\
\hline 1 & PA 801 x AKA 8 & 0.13 & -1.17 & 0.27 & $-3.95 * *$ \\
\hline 2 & PA 801 x PhuleDhanwantary & -0.04 & 0.061 & 0.19 & $-1.86 * *$ \\
\hline 3 & PA 801 x CNA 449 & -0.03 & -0.81 & 0.54 & -0.50 \\
\hline 4 & PA 801 x HD 514 & -0.03 & $2.70 * *$ & -0.66 & -0.45 \\
\hline 5 & PA 801 x DWDa 1402 & -0.01 & -0.43 & 0.64 & $-2.28 * *$ \\
\hline 6 & PA 801 x JLA 794 & -0.07 & 0.41 & $-1.37 * *$ & 0.42 \\
\hline 7 & PA 801 x Digvijay & -0.04 & -0.54 & 0.14 & $4.44 * *$ \\
\hline 8 & PA 801 x G.Cot 23 & 0.09 & -0.21 & 0.24 & $4.18 * *$ \\
\hline 9 & PA $740 \times$ AKA 8 & $-0.10 *$ & $-2.01 * *$ & 0.88 & $1.29 *$ \\
\hline 10 & PA 740 x PhuleDhanwantary & 0.02 & -0.21 & -0.10 & 1.22 \\
\hline
\end{tabular}


Table.1 (Contd...)

\begin{tabular}{|c|c|c|c|c|c|}
\hline $\begin{array}{l}\text { Sr. } \\
\text { No. }\end{array}$ & Genotypes & $\begin{array}{l}\text { Lint index } \\
\text { (g) }\end{array}$ & $\begin{array}{l}\text { Harvest index } \\
(\%)\end{array}$ & $\begin{array}{l}\text { Ginning outturn } \\
(\%)\end{array}$ & Days to maturity \\
\hline 11 & PA 740 x CNA 449 & 0.005 & $-2.44 * *$ & 0.033 & -0.58 \\
\hline 12 & PA $740 \times$ HD 514 & -0.05 & 0.69 & 0.46 & -0.20 \\
\hline 13 & PA 740 x DWDa 1402 & -0.05 & -0.36 & -0.22 & 0.12 \\
\hline 14 & PA 740 x JLA 794 & 0.02 & $-1.79 * *$ & $-1.18 *$ & -1.15 \\
\hline 15 & PA 740 x Digvijay & 0.12 & $3.47 * *$ & 0.85 & 0.53 \\
\hline 16 & PA 740 x G.Cot 23 & 0.06 & $2.67 * *$ & -0.72 & -1.22 \\
\hline 17 & PA 812 x AKA 8 & $-0.18 * *$ & 0.64 & 0.33 & 0.75 \\
\hline 18 & PA 812 x PhuleDhanwantary & 0.04 & $1.47 *$ & 0.47 & 0.015 \\
\hline 19 & PA 812 x CNA 449 & -0.07 & $-1.45 *$ & -0.36 & -0.96 \\
\hline 20 & PA 812 x HD 514 & $0.18 * *$ & 0.67 & $-1.23 *$ & -0.41 \\
\hline 21 & PA 812 x DWDa 1402 & -0.01 & -0.24 & -0.59 & $1.42 *$ \\
\hline 22 & PA 812 x JLA 794 & $0.11^{*}$ & -0.58 & 0.78 & 0.46 \\
\hline 23 & PA 812 x Digvijay & -0.09 & 0.24 & 0.70 & $-1.84 * *$ \\
\hline 24 & PA 812 x G.Cot 23 & 0.02 & -0.74 & -0.10 & 0.56 \\
\hline 25 & PA 809 x AKA 8 & 0.08 & $3.76^{* *}$ & -0.42 & $1.71 * *$ \\
\hline 26 & PA 809 x PhuleDhanwantary & 0.07 & -0.84 & -0.43 & -0.69 \\
\hline 27 & PA 809 x CNA 449 & -0.08 & 0.79 & -0.38 & $1.66^{*}$ \\
\hline 28 & PA 809 x HD 514 & 0.01 & $-2.51 * *$ & -0.60 & 0.045 \\
\hline 29 & PA 809 x DWDa 1402 & -0.01 & -0.98 & 0.67 & -1.12 \\
\hline 30 & PA 809 x JLA 794 & -0.04 & 0.09 & $1.63 * *$ & -0.74 \\
\hline 31 & PA 809 x Digvijay & 0.01 & -0.98 & -0.47 & 0.11 \\
\hline 32 & PA 809 x G.Cot 23 & -0.04 & 0.66 & 0.025 & -0.97 \\
\hline 33 & PA 785 x AKA 8 & 0.06 & -0.08 & $1.29 * *$ & -0.70 \\
\hline 34 & PA 785 x PhuleDhanwantary & -0.009 & 0.84 & -0.22 & 0.89 \\
\hline 35 & PA 785 x CNA 449 & $0.18 * *$ & $2.27 * *$ & $1.78 * *$ & -0.42 \\
\hline 36 & PA 785 x HD 514 & -0.08 & $-1.75^{*}$ & -0.20 & 0.46 \\
\hline 37 & PA 785 x DWDa 1402 & -0.09 & -0.34 & $-2.23 * *$ & $1.79 * *$ \\
\hline 38 & PA 785 x JLA 794 & -0.001 & -0.49 & -0.58 & $2.67 * *$ \\
\hline 39 & PA 785 x Digvijay & -0.05 & -0.61 & -0.44 & $-1.80 * *$ \\
\hline
\end{tabular}


Table.1 (Contd...)

\begin{tabular}{|c|c|c|c|c|c|}
\hline $\begin{array}{l}\text { Sr. } \\
\text { No. }\end{array}$ & Genotypes & $\begin{array}{c}\text { Lint index } \\
\text { (g) }\end{array}$ & $\begin{array}{c}\text { Harvest index } \\
(\%)\end{array}$ & $\begin{array}{c}\text { Ginning } \\
\text { outturn }(\%)\end{array}$ & $\begin{array}{c}\text { Days to } \\
\text { maturity }\end{array}$ \\
\hline 40 & PA 785 x G.Cot 23 & -0.02 & 0.18 & 0.61 & $-2.89 * *$ \\
\hline 41 & PA 832 x AKA 8 & 0.04 & -1.32 & -0.65 & -0.06 \\
\hline 42 & PA 832 x Phule Dhanwantary & -0.06 & 0.44 & -0.31 & 0.36 \\
\hline 43 & PA 832 x CNA 449 & 0.05 & $2.31 * *$ & $-1.08 *$ & -0.77 \\
\hline 44 & PA 832 x HD 514 & -0.10 & -0.69 & 0.24 & -0.89 \\
\hline 45 & PA 832 x DWDa 1402 & 0.06 & -0.09 & 0.16 & 0.77 \\
\hline 46 & PA 832 x JLA 794 & -0.01 & 0.05 & -0.72 & -0.17 \\
\hline 47 & PA 832 x Digvijay & 0.03 & 0.04 & $1.17 *$ & -0.65 \\
\hline 48 & PA 832 x G.Cot 23 & -0.02 & -0.73 & $1.19 *$ & $1.41 *$ \\
\hline 49 & PAIG 346 x AKA 8 & -0.03 & 0.20 & $-1.72 * *$ & 0.96 \\
\hline 50 & PAIG 346 x Phule Dhanwantary & -0.02 & $-1.77 *$ & 0.42 & 0.05 \\
\hline 51 & PAIG 346 x CNA 449 & -0.05 & -0.66 & -0.53 & $1.58 *$ \\
\hline 52 & PAIG 346 x HD 514 & 0.06 & 0.88 & $2.00 * *$ & $1.46^{*}$ \\
\hline 53 & PAIG 346 x DWDa 1402 & $0.11 *$ & $2.47 * *$ & $1.56 * *$ & -0.70 \\
\hline 54 & PAIG 346 x JLA 794 & 0.003 & $2.32 * *$ & $1.45 * *$ & $-1.49 *$ \\
\hline 55 & PAIG 346 x Digvijay & 0.02 & $-1.61 *$ & $-1.95 * *$ & -0.80 \\
\hline \multirow[t]{5}{*}{56} & PAIG 346 x G.Cot 23 & -0.09 & $-1.82 * *$ & $-1.23 *$ & -1.06 \\
\hline & S.E. \pm & & & & \\
\hline & Lines & 0.017 & 0.24 & 0.17 & 0.23 \\
\hline & Tester & 0.019 & 0.26 & 0.18 & 0.24 \\
\hline & Crosses & 0.050 & 0.69 & 0.48 & 0.64 \\
\hline
\end{tabular}


Significance of SCA variances have been reported by Amolik et al.,(1997), Saxena et al., (1998), Pathak and Patel (1999), Deshpande et al., (2003), Nimbalkar et al., (2004), Karademir et al., (2009), Laxman (2010) and Patil et al., (2014) for seed cotton yield and other traits.

\section{Nature of gene action}

The ratio of GCA vs. SCA mean square i.e. predictability ratio for seed index was greater than unity indicating the predominantly additive gene action in the expression of these character. This ratio for boll weight and days to $50 \%$ boll bursting was also very close to unity indicating the presence of additive gene action. The predictability ratio for days to 50 $\%$ flowering, plant height, number of sympodia per plant, number of bolls per plant, seed cotton yield per plant, lint index, harvest index and days to maturity was greater than 0.50 but lesser than 0.80 indicated the equal importance of additive as well as non-additive gene action for these characters whereas the rest of the character possessed lesser predictability ratio than 0.50 indicating the non-additive gene action as the prime cause in the expression of these traits.

\section{Selection of promising parent and crosses}

Lines PAIG 346 and PA 809 and testers CNA 449, AKA 8, JLA 794 and DWDa 1402 appeared to be the best general combines for seed cotton yield and yield contributing characters which may do well in cross combination with other parents. These parents along with high GCA also possessed high per se for yield and yield contributing characters. Line PA 812 was the best general combiner for earliness characters. Selection of the most encouraging cross combination needs high SCA along with high per se and GCA effects of respective parents. The cross combination PAIG 346 X JLA 794, PAIG 346 X DWDa
1402, PA 809 X AKA 8, PA 785 X CNA 449, PA 801X HD 514 were found significantly positive for SCA effects with high mean for seed cotton yield. Whereas, PA 801 X AKA 8 should be further exploited for earliness.

\section{References}

Amolik,V. L., Aher, R. P., Mehtre, S. S. and Darade, R.S.1997b.Combining ability in desi (G. arboreum L.) cotton. ISCI Journal, 22 (1) : 1-6.

Deshpande, L. A., Baig, K. S. and Nagare, Y. P. 2003.Combining ability studies for yield, fibre quality and morphological traits in desi cotton (G. arboreum L.). J. Maharashtra Agric. Univ., 28 (1) : 36-39.

Karademir, C., Karademir, E., Eknc, R., Gencer, O.2009. Combining ability estimates and heterosis for yield and fibre quality of cotton in line $x$ tester design. NotulaeBotanicae, Horti Agrobotanici, Cluj-Napoca.,37(2):222227.

Kempthorne, O. 1957. "An Introduction to Genetic Statistics" JohnWiley and Sons Inc., New York, pp. 453-471.

Kumar K. S., Ashokkumar K., and Ravikesavan R. 2014. Genetic effects for combining ability studies for yield and fibre quality traits in diallel crosses of upland cotton (Gossypium hirsutum L.)Academic Journals.13 (1),pp.119126.

Laxman, S. 2010. Diallel analysis for combining ability for seed cotton yield and its components in desi cotton (G.arboreum L.). j.CottonRes.Dev.,24(1) : 26-28.

Madhuri S., Anita. S., Mashal G. S., and Deshmukh S. B. 2014. Combining ability and heterosis for seed cotton yield, its components and quality in Gossypium hirsutum L. Indian J.Agric.Res.,49 (2):154-159. 
Maisuria, A. T., Patel, J. C., Patel, K. G. and Solanki, B. G. 2006. Study of heterosis and combining ability in GMS based Asiatic cotton (G. herbaceum L.). ISCI Journal, 88-92.

Nimbalkar, R. D., Jadhav, A. C. and Mehetre, S. J. 2004.Combining ability studies in desi cotton ( $G$. arboreum L. and $G$. herbaceum L.). J. Maharashtra Agric. Univ., 29 (2) : 166- 170.

Patel N. N., and Choudhari Pinal. 2015. Combining ability study for yield and its component traits through line $x$ tester mating design in Asiatic (Gossypium herbaceum L.) cotton. J.Cotton Res.Dev.29 (1):19-22.

Pathak, V. D. and Patel, U. G. 1999. Studies on heterosis, combining ability and phenotypic stability in Asiatic cotton G. herbaceum L. Ph.D. Thesis submitted to G.A.U., S. K. Nagar.
Patil, S. S., Magar N. M. and Pawar V. Y. 2014. Heterosis and combining ability for yield and its components in desi cotton (Gossypium arboreum L.) J. Cotton Res. Dev. 28 (2):211-213

Saxena, U., Saxena, M. K., Acharya, V. N. and Ray, N. 1998. Combining ability analysis for seed cotton yield and other characters in $G$. arboreum L. ISCI Journal, 23 (2) : 137-139.

Shakeel, A. Saeed, W. Nazeer, Z. U. Zia and Malik W. 2016. Combining ability analysis and heterotic studies for within -boll yield components and fibre quality in cotton. The Journal of Animal \& Plant Sciences, 26 (1):156162.

Sprague, G. F. and Tatum, L. M. 1942. General versus specific combining ability in single crosses of corn.Agron. J., 34 :923-932.

\section{How to cite this article:}

Chinchane, V. N., D. B. Deosarkar, K. S. Baig and Kalpande, H. V. 2021. Combining Ability Analysis for Seed Cotton Yield and Yield Contributing Traits in Desi Cotton (Gossypium arboreum L.) over the Environments. Int.J.Curr.Microbiol.App.Sci. 10(10): 118-128. doi: https://doi.org/10.20546/ijcmas.2021.1010.015 